\section{El Estado nacional brasileño: desde los antecedentes de su independencia hasta el bicentenario en el 2022}

Recibido: Noviembre de 2016 | Aprobado: Enero de 2017.

\section{Resumen}

La relación histórica entre el Estado y la nación brasileña es un caso único en el continente americano. Dicha relación se inicia como un "estamento burocrático", expresión de un feudalismo portugués trasplantado al Brasil durante la primera época colonial. En 1808 el Estado colonial portugués se instaura en Brasil y después de 1822 con la Independencia surge un imperio independiente cuyo Estado forma la nación. Esta situación continúa en la República hasta la Revolución de 1930 cuyo levantamiento popular impulsa la formación de un nuevo Estado como respuesta nacional a la crisis internacional. Posteriormente, el régimen miliar de 1964 fortalece el Estado en detrimento de la democracia; y en 1985 cuando los civiles retoman el podery democratizan el país, equilibran la relación entre el Estado y la nación. En el siglo XXI se manifiesta un ciclo de auge populista: "el lulismo", el cual colapsa por una severa crisis de corrupción en el año 2016 que desestabiliza la relación entre el Estado y la Nación.

\section{Palabras clave}

Estado/nación - Revolución de 1930Colpe de 1964-Democratización 1985.
Alejandro Mendible Zurita Historiador de la Universidad Central de Venezuela (1974). Magíster en Historia en la Universidad de Wisconsin, EE.UU (1979). Doctor en Historia de las Américas de la Universidad Católica Andrés Bello (1987). Profesor Titular jubilado de la Universidad Central de Venezuela y actual Coordinador de la Maestría en Historia de las Américas (UCV). Ha recibido la condecoración Orden Gran Cruz del Sur del Cobierno de la República Federativa del Brasil (2002), y la Orden José María Vargas, UCV, FHE (1997)

\section{The Brazilian National State: from} the Antecedents of its Independence to the Bicentennial in 2022

\begin{abstract}
The historical relationship between the State and the Brazilian nation is a unique case in the American continent. This relationship beginsasa "bureaucraticestate" expression of a Portuguese feudalism transplanted to Brazil during the first colonial era. In 1808 the Portuguese colonial state was established in Brazil and after 1822 with the Independence emerged an independent empire whose state formed the nation. This situation continued in the Republic until the Revolution of 1930 when a popular uprising promotes the formation of a new State as a national response to the international crisis. Subsequently, the military regime of 1964 strengthens the State to the detriment of democracy; And in 1985, when civilians regained power and democratized the country, they balanced the relationship between state and nation. In the 21st century there is a cycle of a populist boom: "lulismo", which collapses by a severe crisis of corruption in 2016 that destabilizes the relationship between the State and the Nation.
\end{abstract}

\section{Keywords \\ State/nation - 1930 revolution - Coup \\ d'etat of 1985 - 1985 democratization.}




\section{Introducción}

En Brasil, el 7 de septiembre de 2022, se cumplirán 200 años del célebre "Grito de Ipiranga" que selló la independencia nacional de ese país que ocupa hoy la mitad de Sur América. El hecho ocurrió en las placidas márgenes del río de ese nombre, cerca de la ciudad de Sao Paulo, cuando el príncipe Pedro I, entonces regente de Brasil, realizaba el viaje de regreso de supervisar provincias de Minas Cerais y San Pablo y se dirigía hacia la capital Río de Janeiro. En el punto antes señalado recibe la noticia procedente de las cortes de Portugal que lo conminan a retornar a la metrópoli, donde prácticamente podría ser preso. Ante esa situación, opta por exclamar: ¡Independencia o Muerte! La forma teatral asumida por la independencia del Brasil fue diferente a la descolonización de los principales países americanos, donde el triunfo de una batalla decisiva termina con el proceso de confrontación y/o guerras contra la metrópoli, como sucedió en los Estados Unidos y países hispanoamericanos. En Brasil la idea de independencia no fue asumida de inmediato en la conciencia nacional de su población y continuó siendo manipulada a lo largo del siglo XIX por una pequeña élite monárquica que se apropia del Estado nacional con el propósito de formar un gran imperio en los trópicos sudamericanos. Al producirse la independencia, Pedro I, de origen portugués, se convierte en el primer Emperador, pero continúa siendo el heredero del trono de Portugal hasta 1831, cuando a la muerte de su padre Joao VI opta por abdicar al reino del Brasil en favor de su hijo Pedro II, de cinco años de edad, para viajar a Lisboa y encargarse del trono de Portugal.' Este segundo momento creado por la salida del trono de un Pedro portugués y la entrada de otro Pedro brasilero nacido en el palacio real de Río de Janeiro fue considerada por la población como la verdadera independencia.

Se presentaron otros momentos considerados por sus protagonistas como hechos que personificaron la verdadera independencia de un territorio todavía carente de identidad nacional. Entre ellos, casos a nivel regional como la Revolución Pernambucana de 1817, que manifestó un proyecto republicano independiente contra la monarquía colonial gobernante en Río de Janeiro. Uno de sus promotores, el prócer de la Independencia de la Cran Colombia, el pernambucanoy brasileño José Inácio Abreu e Lima, alcanzó el grado de Ceneral otorgado por Simón Bolívar. Éste, después que regresó a su país en 1830, escribió una historia del Brasil en la que califica los eventos de 1817 como la "primera revolución que se hiciera en Brasil". Pensaba que de haber triunfado el movimiento que compartía simpatías con el resto de las causas de Hispanoamérica, eventualmente, Pernambuco y su capital el puerto de Recife se habrían convertido en el centro de referencia republicana para todo el Nordeste del Brasil, concretando la división con el Sur monárquico gobernado por Río de Janeiro. Posteriormente, la creación de la República el 15 de noviembre de 1889 fue considerada por sus promotores como un evento nacional de independencia cuyos antecedentes se remontan a 1870, cuando

1 Al respecto puede verse Boris Fausto, Brasil de colonia a democracia, Madrid, Alianza Editorial, 1995. 
en el periódico La República de Río de Janeiro se pública un manifiesto republicano expresando el deseo de algunos brasileños de igualar al Brasil a los países de América manifestando: "somos de América y queremos ser americanos".

La aceptación del 7 de septiembre de 1822 como la fecha de la Independencia fue el resultado de una elaboración histórica construida por una élite que se apodera del nuevo Estado y controla una inmensa nación inexistente. Para hacerlo actúan las cúpulas regionales integrada por los grandes hacendados esclavistas más interesados en la creación de una nación unida por los intereses del régimen esclavista, que la élite de la corte de la capital Río de Janeiro detentora de la legalidad del poder monárquico, en la persona del príncipe Pedro. ${ }^{3}$

En 1821 el país cumplía trece años con un Estado colonial portugués internalizado como el centro del poder imperial, pero al regresar del rey a Portugal se restablece las funciones metropolitanas y seguidamente desde Lisboa se intenta hacer retrotraer a Brasil a su anterior estatus colonial. Esto determina una reacción cismática del imperio colonial portuguésy la parte luso americana se autonomiza restableciendo la construcción de la nacionalidad como un legado de una élite colonialy creando una unidad político administrativa propia. En este proceso resalta la creación de un Estado reguladory controlador de

2 José Ribeiro Junior, "0 Brasil monárquico em face das republicas americanas", en Mota, Carlos Guilherme (Org.), Brasil em perspectiva, Río de Janeiro, BCD União de Editoras, 2001, p. 160.

3 Miriam Dohllnikoff, "Elites regionais e a construção do Estado nacional", en Itsvan Jancso (org), Brasil: formação do Estado e da nação, Sao Paulo, Hucitec, 2003. un país continental en el cual todavía no existía un espíritu nacional cohesionado. El nuevo monarca, quien representa el poder legal refrendado por la Constitución de 1824, tiene el desafío inicial de consolidar un centro de poder en un territorio tan vasto, de una población de sólo 4.500.000 habitantes, con regiones distantes en las que persisten amplias áreas escasamente pobladas, dominadas por hacendados esclavistas, quienes tienen el poder real y detentan enormes latifundios de características casi autárquicas. Por tal motivo, entre ambos sectores se produce un entendimiento tácito de conciliación que garantiza la aceptación de mantener una estabilidad de gobierno con la finalidad de mantener la unidad territorial. El autoritarismo absolutista fue la ideología dominante durante la construcción del Estado, y, aunque se aprobó una constitución, Pedro I disolvió el congreso para sancionarla por su cuenta dando muestra de su espíritu absolutista. ${ }^{4}$

Desde el Estado, el monarca al frente de una élite que contaba con la anuencia de los sectores del poder regional asume la tarea de formar la nación. Este proceso se ejecuta durante el largo reinado de medio siglo del Emperador Pedro II, un gobernante formado por el Estado para hacerlo de manera correcta y que a su vez consideró a Brasil como su gran pasión. Para realizarlo contó con la estructura patrimonial heredada de la administración colonial portuguesa, a la cual se añadió una expansión considerable del centro burocrático durante la mitad del siglo XIX. En su fecundo reinado, Pedro funda en 1838 el Instituto

4 Sobre la naturaleza del Estado brasilero consultar Fernando Uricochea, The Patrimonial Foundations of the Brazilian Bureaucratic State, California, University of California Press, 1980. 
Histórico Geográfico Brasilero con la intención de contribuir a formar una identidad para el país. El organismo con el cual el Emperador se identificó de manera plena, se convierte en la plataforma de actuación protegida por el Estado de la pléyade intelectual más representativa de su época, entre ellos el historiador Francisco Adolfo Varnhagen (1816-1878), quien crea una narrativa que destaca el contraste entre la monarquía constitucional brasileña y las repúblicas latinoamericanas marcadas por una inestabilidad crónica, ausencia de libertad y guerras civiles. Con este tren de pensamiento se proponía mostrar a Brasil como la única nación civilizada de las Américas. También, el Instituto en la década de 1880, evaluando la crisis de representatividad que enfrentaba el imperio, concibe como estrategia la recuperación de la magnitud del acta de emancipación y repotencia la figura de Pedro I como fundador de Brasil. Como parte de la política en 1888, Pedro Américo, uno de los artistas protegidos y financiados por el Estado, termina el más famoso cuadro sobre la Independencia del Brasil, resaltando la figura de Pedro I como el padre de la patria ${ }^{5}$. Este tramado de actividades queda cortado el año siguiente cuando un golpe de estado, sin apoyo popular, derroca la monarquía y establece un nuevo régimen político republicano bajo las divisas de "Orden y Progreso", pero finalmente incorporaba al Brasil a la marcha de los países americanos. ${ }^{6}$

Bajo los nuevos términos creados, el Estado nacional brasileño toma un tiempo para la

5 Lilia Moritz Schwarcz y Heloisa Murgel Starling, Brasil: Uma Biografía, Brasil, Companhia das Letras, 2015, p. 302.

6 Al respecto ver José Murilo de Carvalho, D. Pedro II, Sao Paulo, Companhia das Letras, 2007. recomposición de los nuevos símbolos en la construcción del imaginario nacional, una mitología que como señala Ceorge Hegel se mueve en la esfera de la fantasía, pero su núcleo interior es racional. ${ }^{7}$ La ideología autoritaria del Estado monárquico es suplantada por los postulados del positivismo con una visión de un orden jerárquico que asume el militarismo en su discurso triunfante. Como la República no tenía un personaje central, un hombre que fuera famoso, ilustre y reconocido por sus virtudes y hazañas, que fuera el centro de unificación o cohesión nacional de la sociedad brasilera, se procede a rescatar el movimiento republicano de 1789. Este movimiento, abortado por las autoridades coloniales en la ciudad de Ouro Preto en Minas Cerais y conocido como la "Inconfidencia Minera", fue influenciado por las ideas del iluminismo, por la Independencia de los Estados Unidos, y mantenía similitudes ideologías a las plateadas por los precursores de la independencia de hispanoamericana. ${ }^{8}$ Los complotados mineros pretendían terminar con el colonialismo portugués, sustituir la monarquía absolutista por la republica liberal, abolir los impuestos, terminar con el mercantilismo e instaurar el liberalismo económico. La figura del militar y político, Joaquim José da Sil Xavier, conocido como Tiradendentes, se había mantenido en la oscuridad hasta 1889, ya que el gobierno de Pedro II lo había mantenido como un traidor a la monarquía. Para rescatarlo, el primer presidente de la República del Brasil, el mariscal Deodoro da Fonseca, lo hace más

7 Georg Hegel, Introducción a la historia de la filosofía, Madrid, Libros Albor, 2000, p.130.

8 Como los planteamientos que Francisco de Miranda sostenía en su exilio en Londres. 
como un héroe religioso que civil. En cuanto a la bandera se crea una nueva, que incorpora el "orden" y el "progreso" como las nuevas divisas nacionales; $y$ en relación al himno se mantiene el del imperio como una victoria popularya que por algún tiempo se tocó la marsellesa, e incluso se llamó a concurso para elaborar uno nuevo. Pero en 1890, en una manifestación militar en la cual se tocó la Marsellesa y otras marchas militares, la multitud pidió que se tocará el viejo himno, y el presidente Deodoro da Fonseca validó el pedido que, según algunos relatos, hizo que muchos de los presentes lloraron. ${ }^{9}$

Los militares tenían una posición autoritaria proclive a crear una sociedad vertical organizada de arriba hacia abajo, conformando un modelo muy diferente al liberalismo compartido por los sectores comerciantes y sectores medios de la población. En el Congreso Constituyente de 1890 la facción civil logra imponerse tomando el modelo de gobierno de los Estados Unidos para imponerlo en la primera Constitución republicana de 1891. Pero no será hasta 1902, cuando el presidente Campos Sales logra articular un pacto políticos de gobernabilidad y alternancia de la primera magistratura nacional entre los gobernadores de San Pablo y de Minas Gerais, que la élite civil desplaza a la militar. El citado pacto político, que reconocía los intereses económicos de la oligarquía del café de Sao Paulo y la ganadera de Minas Grais, se tradujo en un periodo de estabilidad que duró hasta 1930 que ha sido conocido como el de la Vieja República del "café con leche". En este periodo

9 "Sotaques da proclamação", en Revista Veja. http://veja.abril. com.br/especiais/a-republica-e-seus-simbolos/ Consulta realizada el 15 de noviembre de 2016. las elites oligárquicas promueven proyectos de construcción de una nación democrática restringida por los intereses oligárquicos y excluyentes de los grandes sectores populares, pero siguiendo la tendencia transicional y de conciliación creada desde sus orígenes por el Estado brasileño.

Esta situación permite que en 1922 durante la administración del presidente Epitácio Pessoa (1919-1922), cuando se conmemora el festejo del primer Centenario de la Independencia, se produzca una importante reflexión nacional sobre el pasado nacional con el propósito de que los 35 millones de brasileros pudieran uniformar la aceptación de una historia oficial. La cuestión de los orígenes la plantea el primer presidente republicano, el Mariscal Deodoro da Fonseca, en la apertura del Congreso Constituyente de 1890. En los años siguientes se mantiene un largo debate donde los miembros del Instituto Histórico Ceográfico Brasilero ejercen mayor presión persuasiva para validar el "Grito de Ipiranga" formulado por Pedro I como el momento de división de aguas entre lo colonial y lo nacional..$^{10}$ En 1922 se revoca el decreto del Cobierno Provisional que expulsó a la Familia Imperial Brasileña y se toman las previsiones para repatriar los despojos mortales al Brasil. De manera simultánea se emprende un plan integral de modernización de la ciudad de Río de Janeiro y se construye un parque para una exposición internacional conmemorativa del centenario en la cual se resalta el progreso

10 Marly Motta, "1922, 0 Brasil faz 100 anos: a herança portuguesa em questão", en Alda Mourão y Angela De Castro (Coord.), A Experiência da Primera República no Brasil e em Portugal, Portugal, Coimbra University Press, 2014. 
alcanzado por el país. Por otra parte, la reflexión crítica del pasado en función de un presente cargado de contradicciones de todo tipo determina en el plano cultural la exposición contestataria de la Semana de Arte Moderna en el mes de febrero de 1922 en la ciudad de Sao Paulo de la pintora Anita Mafati, que genera todo un movimiento intelectual de repensar el país, como lo manifiesta el Manifiesto Antropológico de Oswaldo de Andrade, uno de los principales líderes intelectuales del movimiento. También, se produce en Río de Janeiro la "Revolta dos 18 do Forte" que inicia la insurrección de "tenentismo", una manifestación de pretorianismo militar."

En 1972 durante la administración del General-Presidente Emilio Carratazu Médici, representante del régimen militar que había tomado el poder mediante un golpe de Estado en 1964, conmemora el sesquicentenario de la independencia nacional y aprovecha para encararlo como un test de popularidad. En el gobierno Médici, el país atravesaba lo que algunos analistas señalaban como un "milagro económico" con crecimiento de $11 \%$; los ingresos recibidos le permiten al régimen militar emprender proyectos de grandes obras de construcciones (la represa de Itaipu, la carretera Trasamazonica, el puente Río-Niteroi, el metro de la ciudad de Sao Paulo, entre otras). Estos cambios eran impulsados por la modernización conservadora del autoritarismo militar con la aspiración de convertir al Brasil en una "Potencia mundial". Esta orientación del

11 Este contexto cultural se puede seguir a través de Oswaldo de Andrade, Escritos Antropófagos. (Selección, cronología y posfácio de Alejandra Laera y Gonzalo Moisés Aguilar), Buenos Aires, Ediciones Corregidor, 2001. crecimiento nacional ocurre a partir del violento viraje de 1964, cuando el ejército toma el poder para cambiar el desarrollo nacional autónomo de tendencia populista, que los golpistas no toleraron por considerar se había distorsionado hacia el comunismo. ${ }^{12}$

Los militares comienzan a redimensionar la historia nacional según sus particulares intereses corporativos a partir del 21 de abril de 1965 . Ese año nuevamente reivindican la figura de Tiradentes, el símbolo de los antecedentes de la insurrección republicana representada por la "Inconfidencia Mineira", declarándolo patrimonio cívico de la nación brasilera y su retrato se coloca en toda la administración pública. Sin embargo, en 1972, considerando la situación imperante de aislamiento del régimen del resto de América Latina por mantener una política exterior proclive al primer mundo, combinada a una estrategia de convertirse una potencia atómica, en la preparación de los festejos de la independencia obvian el carácter panamericano republicano de la celebración para hacer énfasis en la figura del primer Emperador Pedro I. El personaje se presenta en los eventos de la conmemoración del Sesquicentenario como el fundador de la nacionalidady comotal merecedordel culto cívico de la sociedad y de las instituciones brasileras. Para hacerlo el gobierno del Ceneral Médici establece una alianza con Portugal que también se encontraba bajo la dictadura militar del Ceneral Marcelo Caetano para resaltar lo positivo de la colonización portuguesa en Sur Américay se traen los despojos mortales con gran pompa de

12 Janaina Martins, Cordeiro, A ditadura em tempos de milagre; comemoração, orgulhos e consentimento, Río de Janeiro, FGV, 2015. 
D. Pedro I. Éstos, una vez desembarcados en Río de Janeiro, de donde había salido 141 años atrás, emprenden un recorrido de varias semanas por las principales ciudades del país para finalmente ser depositados en el Monumento de Ipiranga en la ciudad de Sao Paulo en una base de cripta.

El propósito del siguiente trabajo es destacar cómola original Independencia del Brasil sintetiza la conjunción de fuerzas disimiles regionales, nacionales e internacionales que determinan la presencia de un Estado actuante como agente mediador que mantiene el equilibrio entre el desarrollo interno del inmenso país y el mundo. En una evaluación de largo alcance histórico, el historiador brasileño Raymundo Faoro señala que Brasil fue gobernado desde la colonia por una comunidad que él denomina de "estamento burocrático", formador del patronato político brasileño determinante de su subdesarrollo. ${ }^{13}$ Sin embargo, esta situación experimenta una alteración determinante a principios del siglo XIX, cuando en 1808 la corona portuguesa se traslada al Brasily la toma como la nueva sede del Estado colonial portugués, situación que cambia trece años después en 1822, cuando el príncipe portugués Pedro I, hijo del rey Joao $\mathrm{VI}$, declara la independencia. A partir de entonces la nueva formación independiente se va enriqueciendo y haciendo cada vez más compleja y profunda en la captación dela identidadnacional brasileña hasta nuestros días, cuando se sigue presentando como uno de los Estados nacionales más originales de los existentes en el continente americano. Teniendo esta visión de referencia desarrollamos

13 Raymundo Faoro, Os donos do poder: formação do patronato político brasileiro, Sao Paulo, Globo, 1996. tres ideas complementarias: la tipicidad de la Independencia del Brasil en el contexto de América Latina, los principales momentos coyunturales solventados por el Estado nacional brasileño a partir de primer centenario de la independencia en 1922 y la situación del Brasil en el siglo XXI, cuando se prepara a conmemorar su Bicentenario después de la superación de la crisis del impeachment que separó de la presidencia a Dilma Rousseff en 2016, terminando con el ciclo populista del lulismo iniciado en 2003.

\section{La ruptura colonial mediatizada}

La independencia del Brasil contrasta por su singularidad y originalidad con procesos similares del resto de países de América Latina. El inmenso país sudamericano pudo conservar la unidad territorial conquistada por el colonizador portugués, siguiendo un proceso evolutivo de transformación no traumático entre el período colonial y el independiente. Asimismo, consiguió separarse de Portugal sin romper el orden social vigente. El factor histórico vinculante lo constituye el largo reinado de 81 años de la dinastía de los Braganzas en Brasil, desde 1808 hasta 1889. Caso único en todo el continente americano, donde una dinastía europea se traslada a un nuevo ambiente tropical, se adapta a las formas sociales existentes y preserva los moldes monárquicos del imperio luso-brasileño y da inicio a una nueva era. Mediado por estas condiciones socio-históricas, la nación brasileña se forma de manera diametralmente diferente al resto de las naciones hispanoamericanas. ${ }^{14}$

14 Newton, Freitas, Los Braganzas, Buenos Aires, Emece Editores, S, A, 1945. 
La llegada de la corona al Brasil fortalece al Estado, y desde ese ente jurídico se va creando la nación que hasta ese momento no constituía un espacio histórico orgánico sino un agregado de regiones dispersas y se inicia un proceso de realización colectiva. En Brasil primero se forma el Estado que la nación, constituyendo un caso atípico bastante diferente al surgimiento de los estados nacionales hispanoamericanos cuyo resultado fue producto de guerras de liberación nacionales. El Estado nacional brasileño aparece después de 1822 como un Imperio sudamericano y el momento de su fundación no se presenta como una ruptura traumática con el pasado colonial por cuanto Pedro I (el primer monarca) continúa dentro de la dinastía portuguesa de los Braganzas. La familia imperial se trasladó a Sur América en 1808 huyendo de la invasión militar francesa y escoltada por la flota de la marina inglesa, llegando al puerto de Bahía el 22 de enero de 1808 , donde el rey Joao VII emite una carta regia declarando la apertura de los puertos al libre comercio. La medida no obstante favorecer los intereses ingleses, significa para los historiadores brasileños el fin del colonialismo económico porque al abolir el mercantilismo que le aplicaba un cepo al desarrollo económico del país entra en una nueva etapa. Posteriormente, en el mes de marzo el rey desembarca en Río de Janeiro acompañado de su esposa Carlota Joaquina, su hijo Pedro y 15.000 miembros de la nobleza portuguesa. Trae también el tesoro real, los archivos de Estado, la imprenta, 60 milvolúmenes de la Biblioteca Real, la gran colección de obras de arte. Río de Janeiro fue designada capital del Virreinato 1763 cuando se instrumentaron las reformas pombalinas aplicadas a todo costo para modernizar el imperio portugués por el favorito del rey d. José I, Senatiao José de Carvalho e Melo (1699-1782) y en 1808 el conde de Arcos era el Virrey quien recibió a monarca. El puerto de Río de Janeiro desempeñaba una función estratégica importante en el desplazamiento del fiel del interés colonial portugués desde el norte hacia el surdonde se tomaba como punto de referencia en el punto de cabotaje hace la apetecida región del Río de la Plata. La ciudad, también, fue el puerto de salida de las importantes remesas de oro de las Minas Cerais, así como el punto principal de ingreso de la trata de comercio esclavo procedente de África y según el historiador brasileño Manuel Oliveira Lima en algunas regiones del interior como Bahía y Minas Cerais estos eran "más numerosos que la población libre". ${ }^{15}$ La ciudad de Río de Janeiro escasamente tenía una población de 80 mil habitantes; de los cuales, por lo menos, 20 mil eran negros esclavos y en 1817 pasó a tener $120.000 .{ }^{16}$ Durante los siguientes trece años que $D$, Joao VI vivió en Río de Janeiro y nombra al conde de Linhares para gobernar la ciudad que paso a ser cosmopolita al recibir las influencias del exterior.

En ese tiempo la antigua colonia ahora metrópoli se trasforma: se instalan en Brasil los ministerios de Guerra y de Relaciones Exteriores, se crea el Banco de Brasil, el Museo Real, una Escuela de Cirugía y Medicina del Hospital Militar, el Jardín Botánico la Academia Militar, la Imprenta Real. También se funda el primer

15 Manuel Oliveira Lima, Formación histórica de la nacionalidad brasileña, Madrid, Editorial-américa, 1918. p.129.

16 Tautphoeus Castilho Bueno, "A corte portuguesa no Brasil", en Revista do Instituto Histórico Geográfico Brasileiro, Brasil, septiembre de 1914, p. 417. 
periódico (Gazeta de Río de Janeiro) y circula el Correio Brasiliense (1808-1822) de Hipolito José da Costa, impreso en Londres como un periódico independiente que contenía críticas a la política portuguesa. Se construye, además, la Quinta de Boa Vista como la vivienda de la familia real, la Escuela Real de Ciencias Artes y Oficios, la Escuela de Bellas Artes con artistas de la misión francesa llegada en 1816, con el arquitecto Grnadjean de Motgney, y los pintores Nicoles Antoni Taunay y Drebet. La ciudad es visitada por científicos y personajes importantes y se convierte en la sede diplomática más importante de América. Fue escenario de la celebración de los actos mortuorios de la reina María, "la loca" (1816) y el matrimonio de Pedro con la archiduquesa María Leopoldina de Habsburgo (1818), hija del Emperador Federico de Austria y la aclamación del rey Joao VI el mismo año. ${ }^{17}$

El estatus de colonia se elevó a centro metropolitano constituyendo un caso único en la historia de América durante el siglo XIX. En esta misma secuencia la presencia de la corona se mimetiza en Brasil, asimilando su nuevo entorno socio cultural. Joao VI, como señala Oliveira Lima, no fue un guerrero ni tampoco fue mártir; se limita a ser un hombre sagaz y bueno en proporción para adaptarse a la idiosincrasia de la sociedad brasileña. Las grandes contradicciones existentes del colonialismo que se habían manifestado en diferentes conjuras y revueltas, siendo la más importante la Já Joaquim José da Silva Xavier, el "Tiradentes" en Minas Gerais en 1781, proponiendo Independencia y Republica,

17 Boris Fausto, Historia do Brasil, Sao Paulo, Editora da Universidade do Sao Paulo, Fundação do Desenvolvimento da Educação, 1995. quedan contrarrestadas con la presencia del rey en Brasil, quien vigoriza el poder colonial. La clase criolla brasileña, a diferencia de la hispanoamericana forzada a tomar una posición de rechazo al poder colonial por las limitaciones que le impone, encuentra en el reino luso brasileño durante su permanencia en Brasil un canal de participación y ascenso social. Por otra parte, la corona portuguesa venía en guerra contra Francia y la continuó en Sudamérica por lo cual en 1809, contando con el apoyo inglés, toma la posesión de Cayena francesa, que le permite por el norte tener acceso al mar Caribe. La apetencia portuguesa por acercarse al Río de la Plata era manifiesta desde 1680, cuando el gobernador de Río de Janeiro Manuel Lobo construye el Fuerte de Sacramento que da origen a la ciudad de Colonia frente a Buenos Aires, con la intención de establecer un límite natural con las posesiones españolas según el impreciso Tratado de Tordesilla. Esta aspiración se potencia al establecer la corona sede en Río de Janeiro en 1808 y se manifiestan dos proyectos expansionistas alternos: el primero, impulsado por la reina española Carlota Joaquina, una absolutista feroz, esposa del rey Joao VII, quien aspira hacer valer sus derechos de sucesión de su padre el rey español Carlos IV y de la situación de hermano menor Fernando VII quien se encontraba preso en Bayona, para crear un reino con sede en Buenos Aires. El proyecto carlotino contó con apoyo parcial del reino portugués y encontró respaldo entre sectores criollos que veían en su realización una forma de separarse de España. Sin embargo, los planes de realización son impedidos por la Junta Central de Sevilla que los rechaza y por las leyes sálicas del reino español 
que no permitía la sucesión femenina. El segundo proyecto expansionista es impulsado por la corona desde los primeros momentos, cuando en 1808 Joao envía un ultimátum a Buenos Aires e invade la Banda Oriental y después intenta dos acciones intervencionistas en la Banda Oriental (hoy Uruguay), la primera en 1811 y la segunda en 1816 después de la derrota de Artigas. La tendencia intervencionista se mantuvo de manera permanente con acciones militares $y$ enfrentamientos de diferentes grados, siendo la más significativa durante la actuación del prócer uruguayo José de Artigas, quien de manera alternativa se enfrentó contra los españoles, los portugueses, los ingleses y los sectores de Buenos Aires. Después de la derrota de Napoleón en 1814 el rey Joao VI decide quedarse en Brasil y en el Congreso de Viena de 1815 propone la creación del reino de Brasil, Portugal y Algarbe con sede en Río de Janeiro. En cuanto al territorio brasileño se decide hacer permanente la ocupación de la Branda Oriental y a tal fin se le convierte en la provincia Cisplatina.

Don Joao $\mathrm{VI}$, entre las muchas cosas que trajo a Brasil, incluyó una excelente colección de Mapas das Cortes que era una verdadera construcción cartográfica con clara finalidad diplomática, como lo demostró de manera fehaciente el negociador portugués Alexander de Cusmao en la negociación con España del Tratado de Madrid de 1750. En esos mapas se encontraban los territorios que abarcaban desde la colonia de Sacramento, en las riberas al Río de La Plata hasta Santos el puerto cercano a Sao Paulo, y abarcando como fondo las regiones de los actuales estados de Río Grande do Sul, Paraná y Santa Catarina, quedando como áreas indefinidas con los españoles los Siete Pueblos de Uruguay donde se habían establecido las misiones de los jesuitas. ${ }^{18}$ La expansión de la colonización lusobrasileña hacia la región del Río de la Plata se incrementa a partir de 1809 cuando desde España se decretó que las colonias americanas dejaban de ser tal para para convertirse en parte integrante de la monarquía. Esto, según John Lynch, motivó el surgimiento de la idea de que no se podía continuar siendo colonia sin una metrópoli e incentivó el comercio interno sudamericano. ${ }^{19}$ Don Joao desde Río de Janeiro dio soporte a este movimiento de penetración interna, el cual incrementó las rutas de animales para el intercambio, amplió la guerra "justa" contra los indios y apoyó la organización de incursiones armadas desde las ciudades de Curitiba y San Pablo dirigidas hacia la región del Plata.

El punto de quiebre del nuevo status-quo colonial que involucraba a Brasil como principal protagonista de los eventos del Imperio portugués desde 1808 se produce en 1820, cuando se produce una revolución liberal en la ciudad de Porto cuyo principal objetivo era el retorno de la corona a su sede natural en Lisboa. La independencia se explica por un conjunto de factores, tanto externos como internos, pero fueron los provenientes del exterior los que imprimieron a los acontecimientos un rumbo imprevisto por la mayoría de actores

18 Mario Clemente Ferreira, "0 Mapa das Cortes e o Tratado de Madrid: a cartografia a serviço da diplomacia", en Revista Varia Historia, vol.23 no.37, Belo Horizonte, Universidade Federal de Minas Gerais 2007, pp. 51-69.

19 John Lynch. América Latina, entre colonia y nación. Barcelona, Critica, 2001, p. 72. 
envueltos en una escalada que pasó de la defensa de la autonomía brasilera a una idea de independencia. En agosto de 1820 estalla en Portugal, en la ciudad de Oporto, una revolución liberal inspirada en las ideas ilustradas. Los revolucionarios procuran enfrentar un momento de profunda crisis en la vida portuguesa. Esta crisis política se produjo por la ausencia del rey y de los órganos de gobierno, acompañada por una crisis económica resultante en parte de la libertad de comercio de la cual se beneficiaba Brasil y de una crisis militar, consecuencia de la presencia de oficiales ingleses en los altos puestos del ejército y de la inmovilidad en el ascenso de los oficiales portugueses en sus promociones. En la ausencia de D. Joao, Portugal fue gobernada por un consejo de regencia presidido por el Mariscal inglés Beresford, quien después de finalizada la guerra se convierte en el comandante del Ejército Portugués. La revolución portuguesa de 1820 tenía aspectos contradictorios para los brasileños. Podía ser definida como liberal por considerar la monarquía absoluta como un régimen atrasado y opresivo y por dar vida a órganos de representación, como el caso de las Cortes. Al mismo tiempo, al promover los intereses de la burguesía lusa e intentar limitar la influencia inglesa, pretendía hacer de Brasil regresar a su subordinación colonial de Portugal.

La situación anterior determinó el regreso de JoaoVIaPortugal presionado porellevantamiento de las tropas acantonadas en las ciudades de Belén y Bahía apoyando el movimiento revolucionario y de las manifestaciones populares ocurridas en Río de Janeiro. El rey se embarca de regreso en abril de 1821 acompañado de 4 mil portugueses y llevando de regreso muchos de los bienes que trece años antes había traído, entre ellos los cofres reales que dejaban a Brasil en dificultades económicas. La sociedad se encontraba escindida entre el sector más protagónico y dominante integrado por aquellos que apoyaban el retorno constituido por una "facción portuguesa" formada por los altos mando militares, burócratas y comerciantes interesados en subordinar a Brasil a la Metrópoli, y otro menos estridente que representaba a los sectores criollos, el "partido brasileño", constituido por los grandes propietarios rurales, los burócratas y miembros del poder judicial nacidos en Brasil, que más que un partido constituía una corriente de opinión. Las articulaciones políticas se hacen en este periodo a través de las lógicas masónicas y Pedro I, quien había sido dejado por su padre como príncipe regente se convirtió en una figura central de la orden en Brasil. El príncipe encargado confronta durante 1821 un desafío frontal para su estabilidad de gobernante producto de medidas tomadas por las Cortes de Portugal, las cuales sin la presencia de los delegados brasileños acordaron pasar la dependencia de las gobiernos provinciales de Río de Janeiro para ser asumida por Lisboa, lo que acarreaba la desintegración de la unidad brasileñay un regresoa la subordinación colonial. Las cortes intentaron, también, revocar los acuerdos comerciales con Inglaterra que eran del interés tanto de los ingleses como de los grandes propietarios rurales brasileños y de los consumidores urbanos. Todo ello asumiendo una actitud prepotente que al soliviantar las fuerzas profundas del nacionalismo brasileño llegó a un momento de clímax cuando se intentó forzar el regreso de Pedro a Portugal. Esto 
coincidía con los esfuerzos del "partido brasileño" de conseguir la permanencia del príncipe en Brasil, lo cual se da con el solemne "día do fico", ocurrido el 9 de enero de 1822, que señala la escogencia de un camino sin retorno. A partir de allí se precipita cuando Don Pedro obliga a las tropas portuguesas a retirarse de Río de Janeiro al negarse a jurarle fidelidad y forma un nuevo ministerio compuesto de portugueses pero cuya jefatura la hace recaer en un brasilero, José Bonifacio de Andrade e Silva. Este es considerado como el "Patriarca de la independencia", el más notable agente de la emancipación desde el punto de vista individual y desde su posición de representante de las aspiraciones brasileñas. Estudió y fue profesor destacado en la Universidad de Coimbra y participó en la lucha de resistencia contra la ocupación francesa en Portugal. Regresa a Brasil en 1819 y, después de participar por corto tiempo en el gobierno provisorio del Estado de Sao Paulo (donde había nacido en la ciudad de Santos en 1763), pasa a convertirse en el principal consejero de Pedro I, a quien apadrina para convertirse en la masonería. Desempeña un rol predominante en la formación del nacionalismo brasileño, defendiendo un gobierno parlamentario, la abolición del tráfico africano de esclavos, la creación de escuelas y la práctica de la cultura física para la juventud; también fue el primer canciller del imperio independiente. $^{20}$ En ese cargo, un mes antes del Grito de Ipiranga, envió el Manifiesto del 6 de agosto de 1822, considerado como el primer documento dirigido por Brasil a las naciones del mundo explicándole la situación de D, Joao

20 Joao Alfredo dos Anjos, José Bonifacio, primeiro Chancele do Brasil, Brasilia, fundação Alexander de Gusmão, 2008, p.83.
VI como tutelado de las cortes portuguesas y anunciando la fundación del Imperio brasileño, por cuanto los brasileños no admitirían regresar al régimen anterior de sumisión a los intereses de Portugal. En esa oportunidad José Bonifacio comparte la idea de americanidad y de mantener la unidad entre las nuevas naciones libertadas; se muestra partidario de que el espacio lusobrasileño se mantuviese como una unidad formando parte de una confederación de todos los Estados libres de América, a fin de contrabalancear la confederación europea. ${ }^{21}$ Este punto lo había mantenido con anterioridad cunado el 24 de mayo nombra un cónsul en Buenos Aires con el encargo de convenir en una alianza entre los países americanos en defensa de su libertad e independencia amenazada por las "pretensiones de Europa" y autorizando al mismo representante a proponer al gobierno de Buenos Aires un tratado de alianza ofensiva y defensiva y asegurando que "el Brasil grande, rico y poderoso sólo procuraba de vecinos prósperos y venturosos para comerciar y defenderse con ellos".22

Esta aspiración se cumplió logrando el espacio colonial lusoamericano mantenerse como una unidad independiente a diferencia de lo ocurrido con los otros espacios coloniales europeos en América. Esto, según Oliveira Lima, fue posible por cuanto, "Brasil pudo escapar a la crisis prolongada que atravesaron los otros pueblos latinoamericanos, gracias a la monarquía, que

21 En este punto representa la visión brasileña del panamericanismo. Este visón se complementa con la doctrina Monroe de formulada por los Estados Unidos en 1822 y la de Simón Bolívar en el Congreso de Panamá de 1824.

22 Octavio Tarquínio de Sousa, José Bonifacio, Río de Janeiro, Livraria José Olympio, 1974, p. 141. El cónsul designado fue Manuel António Correia da Câmara. 
es la autoridad sin la tiranía, la fuerza sin la violencia, la moral sin la hipocresía y la libertad sin la indisciplina".23

Este período de la historia brasileña marca el rumbo a seguir por el país independiente y su conocimiento es determinante para entender el Brasil contemporáneo, así como establecer las diferencias con el resto de América Latina ${ }^{24}$. Sin embargo, los principales acervos de fuentes históricas se encuentran en el Archivo Histórico de la Torre de Tombo en Lisboa (el equivalente al Archivo de Sevilla), el archivo imperial en la ciudad de Petropolis cerca de Río de Janeiro, en la Biblioteca Nacional de Río de Janeiro y la Biblioteca del Instituto Histórico Ceográfico Brasileño en la misma ciudad.

\section{Orden y progreso: la conciliación nacional después de 1922}

La revolución de 1930 establece en Brasil el choque frontal mediado por la grave crisis económica internacional entre el país rural agro exportador representado por el latifundio semifeudal asociado al imperialismo ${ }^{25}$ y a los intereses volcados hacia el mercado interno y los intereses de una "burguesía" en formación en alianza con las aspiraciones de las clases medias y los movimientos militares, representado por los "tenientes". El desenlace del conflicto

23 M. Oliveira, "Formación histórica de la nacionalidad brasileña", p. 145 .

24 Sobre este período fundacional ver Ron Seckinger, The Brazilian Monarchy and the South American Republic 1821-1831, Baton Rouge, Louisina State University Press, 1984.

25 El canciller Río Branco entre 1902 y 1912, estimando como la exportación del café se orientaba hacia los Estados Unidos, en su mayor medida, establece una "alianza tacita" con este país. dio inicio a un país urbano e industrializado como una respuesta nacional a la gran crisis del sistema internacional acarreada por la quiebra de la bolsa de Nueva York. La hecatombe no era previsible a principios de 1929 cuando el presidente Washington Luis gobernaba con relativa tranquilidad desde Río de Janeiro, pero su determinación de tratar de imponer como su sucesor en la primera magistratura al gobernador del Estado de Sao Paulo, Julio Preste, determinó una fuerte fisura entre las elites de los grandes Estados, resquebrajando el pacto de gobernabilidad acordado desde 1902 entre los estados de San pablo y Minas Gerais, que le daba estabilidad al periodo de la Vieja República. La actitud de Washington Luis impulsó a los políticos mineros que representaban la oligarquía ganadera a no sentirse obligados a apoyar las pretensiones de reposición gubernamental de los políticos de la oligarquía del café de Sao Paulo y sentirse liberados para buscar nuevas alianzas, la cual consiguen con los sectores políticos gauchos, de Río Grande do Sul. Este Estado había sido marginado del pacto gubernamental pero, con el incremento de su economía en las primeras décadas del siglo XX $y$ con el aumento del comercio internacional del sector ganadero, pugnaba por un mejor reconocimiento político. A mediado de 1929, después de varias conversaciones, se organiza una coalición opositora para enfrentarse al gobierno de Washington Luis, encabezada por Cetulio Vargas, como candidato a la presidencia, el joven gobernador de Río Crande do Sul y Joao Pessoa, para la vicepresidencia, gobernador del estado de Paraíba, con lo que se buscaba la adhesión de los votantes de la empobrecida 
región del nordeste. La nueva propuesta creó la Alianza Liberal como el aparato de propaganda electoral de las elecciones que recorre el país identificándose con las aspiraciones de las clases dominantes regionales no asociadas al núcleo cafetalero (el principal sostén del Estado) y tenía por objetivo sensibilizar la lucha de las clases medias. Estos sectores compartían con el gobierno que los esquemas de valorización del café no se debían mantener sólo en nombre de la ortodoxia financiera, pero discordaban con el presidente Washington Luis en la situación las mejoras de los trabajadores de menos ingresos y de las mujeres, que él consideraba era, "una cuestión de la policía". En el mes de octubre de 1929 estalla la crisis económica internacional la cual de inserta de manera dramática en la formación de Brasil en momentos en que la producción de café para 1930 fue muy alta, en contrapartida a lo esperado, empujando una crisis imposible de compensar ante la caída de los precios con la ampliación del volumen de venta y rompiendo toda posibilidad de entendimiento entre el sector cafetalero y el gobierno federal.

Después de la Revolución se proyecta la conducción de Cetulio Vargas al frente de la presidencia del Brasil por quince años sucesivos hasta 1945. Vargas se convierte en el gran articuladordel nuevo procesodecambio nacional, cambiando su tradicional modo de inserción en el sistema internacional de agro exportador a otro con acento en la industrialización. Para implementarlo, Vargas rechaza el sistema federal imperante durante la Vieja República y fortalece la centralización, mediante lo cual, reorienta la tradicional ideología autoritaria del
Estado brasileño, apelando al nacionalismo para formar una nueva élite de Estado con acentuado acento político. Con gran habilidad realiza una manipulación del pasado, apelando a los mitos fundacionales nacionales para fortalecer su situación política en el presente. Expresa en varias oportunidades que no se le permitiría a nadie dudar de la grandeza del Brasil; hace uso simbólico del himno y de la bandera nacional; funda el Museo de la Inconfidencia para honrar a Tiradentes como héroe de la República, y a la vez el Museo Imperial, Petropolis, para honrar los aportes de la monarquía; y emplea las figuras del Duque de Caxias, el padre del ejército", el Barao Rio Branco, el canciller de las fronteras nacionales y Tiradentes en sus contundentes discursos contra el comunismo después de la insurrección de $1935 .{ }^{26}$

La nueva élite surge de la alianza entre sectores sindicales, sectores medios y burguesía industrial que forma un nuevo polo de poder que se fortalece preferentemente durante el período del Estado Novo (1937-1945). Se consolida una burocracia de Estado que le permite al país aprovechar la confrontación bélica para fortalecer su posición en las negociaciones internacionales con las potencias involucradas en la contienda y que terminan con una alianza estratégica con los Estados Unidos. En este contexto avanza un modelo económico sustentador de un desarrollo nacional autónomo, que a partir del fin de la segunda guerra mundial entra en un período democrático dentro del marco de la constitución liberal de 1946.

26 Sobre esta importante coyuntura histórica ver Boris Fausto, $A$ Revolução de 1930: historiografía e história. Sao Paulo, Companhia das Letras, 1997. 
El período democrático se inicia con el General Dutra, lo sucede después de las elecciones de 1950 Getulio Vargas, quien trata de reponer el modelo nacionalista aplicado durante la guerra, pero en esta oportunidad al aplicar tácticas populistas su intento choca contra la realidad creada por un contexto muy diferente a nivel nacional e internacional. En Brasil se forma un fuerte frente conservador y en el plano internacional la guerra fría ha dado forma a nuevos esquemas de poder; todo ello lo conduce a encontrarse aislado $y$, con poco margen de maniobra, opta por el suicidio en agosto de 1954. Sigue una década de un varguismo sin Vargas con una fuerte carga de populismo en la cual sólo el presidente Juselino $\mathrm{K}$ logra completar su periodo y ejecutar una obra de gran aliento histórico con la fundación de Brasilia, como la nueva capital. Pocos años después, la democracia se interrumpe de manera abrupta con el golpe de estado militar que derroca al presidente Joao Goulart en 1964.

Desde mediados de la década de 1950 aumenta de manera significativa la inversión extranjera y asciende el capital multinacional creando una dinámica interna tendiente a la consolidación de un polo de presión interno que influye sobre las instituciones establecidas representantes del modelo de desarrollo económico nacional independiente. De esta manera el Estado nacionalista de desarrollo creado por el varguismo entra a partir de 1960 en un proceso de internalización por la formación de un capital multinacional y asociado que presiona por un cambio de rumbo del proceso de desarrollo nacional. El creciente nuevo sector económico internacional en connivencia con sectores políticos brasileños presiona por la creación de un nuevo Estado que represente los sectores de un capitalismo asociado. Se produce un agudo proceso de desestabilización a partir de la renuncia intempestiva del presidente Janio Quadros, en agosto de 1961, cuando la nueva dinámica política determina que los sectores garantes de la estabilidad del varguismo y la base de apoyo político del nuevo presidente encargado Joao Goulart, integrado por los partidos PTB y el PSD, se debilitan, como producto del traslado que el segundo hace del centro a la derecha acercándose a la oposición conservadora liderado por el UDN. Se produce una politización de los sectores empresariales ante el agravamiento de la crisis económica, compartida por los altos mandos del ejército para salvar del caos al país. En medio de esta situación se acelera el desgaste del presidente Coulart; algunos lo ven como un líder comunista que pretendía implantar en Brasil una república sindical; otros como un político demagogo que por ser un gran estanciero millonario no estaría comprometido con la reformas del país que el mismo proponía; y también se encontraban los que lo consideraban un caudillo autoritario, o como débil, incompetente y desesperado en ejercicio de sus funciones. ${ }^{27}$ El 31 de marzo de 1964 se produce un golpe de estado que derroca al presidente Goulart, pone fin al régimen democrático e instituye la dictadura en Brasil, recogiendo el nuevo contexto que adoptaba la guerra fría en América Latina después del triunfo de la Revolución cubana y la injerencia del comunismo en el Caribe.

27 Jorge Ferreira, Joao Goulart: uma biografia. Rio de Janeiro, 2011. 
Los militares fueron los actores protagónicos del evento de 1964 y por 21 años lograron canalizar fuerzas heterogéneas, reuniendo corrientes políticas conservadoras, liberales y nacionalistas en un consenso "anticomunista", el cual impulsó un modelo de "modernización conservadora”. En este período el gobierno militar brasileño, actuando como el regente del Estado, manifestó su ambición de convertirse en una potencia mundial y mantuvo una política hacia el primer mundo obviando a los países latinoamericanos. En el plano interno se suprimieron los derechos civiles fundamentales mediante actos instituciones llegando durante la presidencia del general Artur de Costa e Silva, bajo el pretexto de la "seguridad nacional", a decretar el A.l-5 que cerraba el Congreso Nacional y suspendía el habeas corpus. Para los efectos del presente trabajo destacamos lo señalado con anterioridad de la celebración del sesquicentenario de la independencia en 1972 durante el gobierno del general Emilio Carratazu Médici identificado con el momento de euforia del régimen por el alto crecimiento económico del país que llegaba a un PTB de hasta $11 \%$, en lo que se consideraba un "milagro económico brasileño". En estas condiciones el gobierno militar reedita el mito fundacional del Brasil como el "destino manifiesto" luso brasileño en los trópicos sudamericanos destinado a convertirse en potencia mundial.

El último reacomodo importante del Estadonacional brasileño en el siglo XX se produce una vez que la sociedad civil logra la democratización en 1985. A partir de la recuperación del gobierno por los civiles se crea un nuevo tipo en las relaciones civiles militares y de negociación para el regreso de los militares a los cuarteles. La nueva situación se plasma en la constitución de 1988 que en su preámbulo señala: "nosotros, representantes del pueblo brasileño, reunidos en Asamblea Nacional Constituyente para instituir el Estado Democrático, destinado a asegurar el ejercicio de los derechos sociales e individuales, la libertad, la seguridad, el bienestar, el desarrollo, la igualdad y la justicia como valores supremos de una sociedad fraterna, pluralista y sin preconceptos, fundada en la harmonía social y comprometida, en el orden interno e internacional, con la solución pacifica de las controversias, promulgamos sobre la protección de Dios". ${ }^{28}$ La lucha nacional por la recuperación democrática concientizo a la sociedad brasileña valorando el derecho la ciudadanía y que esta causa no era solamente nacional sino compartida con los otros pueblos del continente. Esto motivo el acercamiento con Argentina y el inicio en 1991 del Acuerdo de Mercosur como un proyecto de entendimiento para impulsar el surgimiento de una nueva realidad geohistórica sudamericana.

\section{Siglo XXI: del impeachment al nuevo orden y progreso}

Durante la primera década del siglo XXI se crea un nuevo contexto internacional con efectos importantes en el desarrollo geo-histórico de América del Sury en el impulso del protagonismo de Brasil en el continente. En primer lugar se resiente el paradigma de América Latina, cuando el 1 de enero de 1994 entra en vigencia el Tratado

28 Constitución Federativa del Brasil de 1988 en http://pdba. georgetown.edu/Constitutions/Brazil/esp88.html Consultado el 20 de noviembre de 2016. 
de Libre Comercio de América del Norte (TLCAN) con la inclusión de México. La nueva posición de ese país le resta influencia a su tradicional nacionalismo contra el intervencionismo norteamericano vigente en la región desde la Revolución mexicana de 1910, e impulsa a Brasil a llenar un espacio protagónico que tradicionalmente evadió. El 11 de septiembre de 2001, después del impactante ataque terrorista a los Estados Unidos, se produce un drástico viraje de los objetivos geopolíticos de la potencia del Norte orientados hacia el Medio Oriente, atacando militarmente a Irak y Paquistán. Esta situación dejó al margen a la América del Sury al Brasil como áreas de paz y de no confrontación. Por otra parte, en 2008 estalla la crisis económica en los Estados Unidos, conocida como la "burbuja inmobiliaria" creada por el banco Leman Brothers y cuyos efectos se expandieron a las economías de Europa y Japón, creando una grave crisis del sistema capitalista mundial. Esta situación repercute en Brasil de una manera diferente a lo que ocurrió en la gran crisis de 1930, cuando su economía cafetalera colapsa; en esta oportunidad el gobierno brasileño impulsa un programa anti-cíclico de expansión económica, ampliando el gasto público contando con el alto aporte de la inversión de la economía China en su ascenso como potencia mundial.

Los cambios internacionales crean una coyuntura favorable en Sur América para cohesionarse como una región de unidad geoeconómica y superar la tradicional disgregación existe el pasado siglo entre sus diferentes Estados nacionales integrantes. La sudamericanización gana viabilidad por cuanto se alcanza una nueva realidad democrática legitimada por cuanto todos los mandatarios son electos en comicios nacionales. Esta situación se pone en manifiesto en el 2000 cuando el presidente Fernando Henrique Cardoso de Brasil, (quien realizaba la hazaña histórica de estabilizar la incontrolable economía inflacionaria mediante la aplicación del "Plan Real"), para festejar los 500 años del descubrimiento de su país invita a los 12 presidentes del continente a asistir a Brasilia. La cumbre de mandatarios termina con la aprobación de convertir el continente en un espacio común regido por una conducción democrática. La cooperación intergubernamental se objetiva en mayor grado en los países de Mercosur a partir de 2003, cuando Lula toma la presidencia del Brasil y se reúne con el presidente argentino Néstor Kirchner y acuerdan combatir las políticas neoliberales del Acuerdo de Washington. En cuanto a las cumbres presidenciales tienen un ciclo virtuoso que por primera vez en la historia de la región concretan la fundación de UNASUR como una entidad supranacional.

En Brasil, a partir del 2003, se inicia un quiebre del centro político estabilizador del país alcanzado en 1994 cuando fue electo presidente Fernando Henrique Cardoso del Partido Social Democrático Brasileño. Esta organización y el Partido de los Trabajadores, creados ambos después de conquistada la democratización en 1985, se disputaban el espacio de la izquierda ya que cada una de estas dos organizaciones se convertía en el segundo turno de las elecciones presidenciales en marco de referencia para la ubicación de los numerosos partidos brasileños 
por ocupar el centro político de derecha o izquierda. ${ }^{29}$ En el 2003 la elección de Luíz Inacio Lula da Silva del PT impulsa un viraje del país hacia la izquierda con la pretensión de capitalizar el clivaje electoral nacional en procura de crear un estado de bienestar de gran inclusión social, creando el fenómeno del lulismo. El lulismo manifestó su voluntad política de refundar el Brasil y establecer una ruptura diametral con el pasado nacional, contando en buena medida con la coyuntura favorable creada en la región señalada anteriormente. Además aspiró a que el país se convirtiera en el centro geopolítico de la nueva tendencia sociopolítica adoptada por el Mercosur. Consecuentemente, Lula en su discurso inaugural manifiesta que Sudamérica sería prioritaria en la política exteriory durante su gestión presentaba al Brasil como la locomotora de la nueva orientación del continente.

Lula en sus dos periodos de gobierno entre el 2003 y 2010 alcanza un alto nivel de aceptación nacional. Un estudioso del periodo, el sociólogo brasileño André Singer, señala en su libro Os sentidos do Lulismo, que a partir de 2003 se produjo una innovadora orientación gubernamental que le permitió contar con la mudanza de la coyuntura económica internacional, de adopción de políticas para reducir la pobreza y para la activación del mercado interno sin confrontar el capital. Esta situación habría producido en asociación con la crisis creada por el escándalo político - mediático del acto de corrupción administrativa Ilamado "mensalao", lo cual creó un realineamiento electoral que se cristalizó para ganar un segundo periodo

29 En el 2016 en Brasil existen 33 partidos diferentes. presidencial. El surgimiento de una base lulista a su vez proporcionó al presidente Lula mayor margen de maniobra posibilitando acelerar una implantación del modelo de "diminución de la pobreza manutención del orden". ${ }^{30}$ De esta manera Lula desde la presidencia emprende un agresivo plan de recuperación social y logra rescatar 35 millones de brasileños de los niveles de pobreza, al tiempo que Brasil alcanza la séptima posición entre las economías industrializadas.

Lula y el PT montados en una onda de la refundación del Brasil pensaron que su presente promisor se proyectaría sin mayores alteraciones hacia el futuro. Así, en 2009, el presidente Lula le encomienda al ministro de Asuntos Estratégicos (SAE) Samuel Pinheiro Guimaraes la elaboración de un estudio de factibilidad sobre las posibilidades de desarrollo de Brasil para el 2022 cuando el país arriba a su bicentenario de independencia. La aspiración era dibujar un país gran potencia mundial dotada de un estado de bienestar plasmado en una pirámide grandemente abultada en el medio por la existencia de una clase media con posibilidades de alto nivel de consumo. En el 2010 cuando Lula no podía optaraun tercer mandato porimperativo de la constitución, empleó su liderazgo personal para nominar a Dilma Rousseff como su sucesora en la presidencia. La nueva gobernante, la primera mujer al frente de la presidencia de la república del Brasil, continúa con el impulso favorable del modelo creado por Lula, pero no contando con el carisma de su protector, por lo cual bajó la imagen del país en América Latina,

30 André Singer, Os sentidos do lulismo. Reforma gradual e pacto conservador, Sao Paulo, Companhia das Letras, 2011, p.13. La traducción es nuestra. 
aunque continúa el protagonismo internacional y en particular en los países del llamado Brics. Con Dilma se produce el reconocimiento deportivo del país en dos eventos que elevan el ego gubernamental, la copa del mundial de futbol de 2014 y la selección de la ciudad de Río de Janeiro como sede de las olimpiadas mundiales en 2016. En la preparación de ambos eventos se produce una millonaria inversión para renovar la infraestructura deportiva y transporte con el fin de organizar los eventos

Dilma mantenía una alta aceptación hasta mediados de 2013 cuando iniciaron los primeros señalamientos sobre la situación económica y eventuales indicios de corrupción administrativa. Las nuevas condiciones del país fueron deteriorando la imagen presidencial $y$ ante el alza de las tarifas del transporte se produjeron protestas generalizadas en varias ciudades del país. Sin embargo, en la elecciones de 2014 Dilma pudo retener la presidencia por muy poco margen, en una cerrada competencia contra el candidato opositor del PSDB, Aesio Neves, quien la denunció por mantener un esquema de corrupción en Petrobras, la primera empresa pública nacional. Las cosas empeoraron para Dilma a partir del 2015 cuando se hacen públicas las denuncias de "pedaladas fiscales" consistentes en maquillar las cuentas nacionales con la finalidad de mostrar una situación irreal de la economía nacional y ganar las elecciones. El caso tipificado como estelionato desató un encrespamiento del debate político que experimenta un escalamiento significativo después de la separación del partido PMDB del gobierno. Y, a partir del 2 de diciembre de
2015 el Presidente de la Cámara de Diputados Eduardo Cunha (quien posteriormente resulta separado del cargo en el mes de septiembre de 2016 y pasado a prisión por casos de corrupción), da inicio al proceso de impeachmente incriminando a la presidente Dilma por el "delito de responsabilidad", ${ }^{31}$ juicio que después de seguir todos los tramites e instancias requeridas termina el 17 de abril de 2016 con la separación de Dilma de su cargo. El desenlace del traumático proceso es calificado por Dilmay seguidores como un "golpe de estado" blanco, y el hecho genera gran repercusión en los medios informativos nacionales e internacionales.

El declive político del Iulismo fue acompañado de la elevación de la crisis y aguda recesión, cuestión que no cesó con el desplazamiento de Dilma de la presidencia por Michel Termer en su sustitución y sigue mermando las aspiraciones de Brasil como potencia regional y mundial. La falta de legitimidad y la ruptura del consenso político nacional le asestaron un duro golpe al proceso de crecimiento económico. Esta situación se ve estimulada por un fenómeno inédito en la historia del Brasil consistente en la automatización y fortalecimiento de la justicia por encima de los otros poderes públicos, en particular de la operación "lava jato" iniciada en marzo de 2014 contra el caso de corrupción de la compañía estatal Petrobras, ${ }^{32}$ cuya incidencia logró desestabilizar la

31 Según la Constitución de 1989, en el artículo 56 relacionado con la competencia privativa del Senado Federal, se indica como el primero: "procesar y juzgar al presidente y al vicepresidente de la república en los delitos de responsabilidad"

32 La acción "lava-jato" tiene implicaciones para gobiernos aliados del lulismo en la región. como el chavismo en Venezuela. Ver: "América Latina sufre el impacto del Lava-Jato", El Nacional, Caracas, lunes 12 de diciembre de 2016. 
clase política nacional y convertirse en el hecho noticioso y político más importante del país, sirviendo de marco del protagonismo alcanzado durante sus actuación los jueces Sergio Moro y Rodrigo Janiot. ${ }^{33}$ La operación contra la corrupción alcanzó los más altos niveles de la vida nacional, entre ellos a empresarios, políticos de todos los partidos, a los presidentes de la Cámaras de Diputados y del Senado, e incluso al ex presidente Lula. Los analistas políticos de la situación brasileño coinciden en señalar que el enfrentamiento entre los poderes públicos es la más grave de las ocurridas en la historia del país. Esta situación abre un compás de espera hasta que se calme el encrespado panorama político y la sociedad brasileña se pronuncie para crear una nueva base de legitimación o pacto político nacional que en final de cuentas será el reacomodo del Estado nacional que el 7 de septiembre de 2022 llegará a su bicentenario, mostrando en qué medida será su influencia como el país del futuro.

\section{Bibliografía}

\section{Fuentes primarias}

Folha de Sao Paulo

Globo

Jornal do Brasil

Revista Veja

El Nacional

33 El profesor Moniz Bandeira, en entrevista al periódico Jornal de Brasil del 3 de diciembre de 2016, señala que "Moro e Janiot atuam com os Estrados Unidos contra o Brasil". Según Bandeira en su actuación desestructuran, paralizan y descapitalizan las empresas brasileras, estatales y privadas como Odebrecht que compite en el mercado internacional, América del Sur y África. "Moniz Bandeira: "Moro e Janot atuam com os Estados Unidos contra o Brasil", Jornal do Brasil, diciembre 3 de 2916.

\section{Fuentes secundarias}

Anjos, Joao Alfredo dos, José Bonifacio, primeiro Chancele do Brasil. Brasilia, Fundacao Alexander de Gusmao, 2008.

Bueno, Eduardo, Brasil: uma história. A incrivel saga de um país. Sao Paulo, Atica, 2003.

Cabral, Filho, O mundo latino é a era de megestado (http://wwwpublico.es/internacional/88550/ brasil/superpotencia/america)

Calmon, Pedro, Historia Social del Brasil. Sao Paulo, Companhia Editora Nacional, 1957.

Calogeras, ]. Pandia. Fomacao histórica do Brasil. Rio de Janeiro, Biblioteca do Exército, 1957.

Carvalho, José Murilo. D. Pedro II. Sao Paulo, Companhia das Letras, 2007.

Cordeiro, Janaina Martins, A ditadura em tempos de milagre: comemoração, orgulhos e consentimento. Rio de Janeiro, FCV, 2015.

Castilho bueno, Tautphoeus, "A corte portuguesa no Brasil", en Revista do Instituto Histórico Geográfico Brasileiro. Septiembre de 1914.

Cervo, Luis Amado y Bueno, Clodoaldo, Historia da política Exterior do Brasil. Brasilia, Editora Universitaria de Brasilia, 2008.

Clemente Ferreira, Mario. "O Mapa das Cortes e o Tratado de Madrid: a cartografía a servicio da diplomacia", en Revista Varia Historia Belo Horizonte vol. 23, no 37: pp 51-69, Jan/Jun. 2007. 
Oswaldo de Andrade, Escritos Antropófagos. (Selección, cronología y posfácio de Alejandra Laera y Conzalo Moisés Aguilar), Buenos Aires, Ediciones Corregidor, 2001.

Dohllnikoff, Miriam. Elites regionais e a construcao do Estado nacional en, JANCSO, Istvan (org) Brasil: formacao do Estado e da nacao. Sao Paulo, Hucitec, 2003.

Dreifus, Rene Armand, A conquista do Estado. Petropolis, Voces, 1986.

Faoro, Raymundo, Os donos do poder: formacao do patronato político brasileiro. Sao Paulo. Clobo, 1996.

Fausto, Boris, Brasil de colónia a democracia. Madrid, Alianza Editorial, 1995.

A Revolucao de 1930: historiografía e história. Sao Paulo, Companhia das Letras, 1997.

Historia do Brasil, Sao Paulo, Editora da Universidade de Sao Paulo. Fundacao do Desenvolvimento da Eucacao, 1995.

Ferreira, Jorge, Joao Goulart: uma biografia. Rio de Janeiro, 2011.

Freitas, Newton, Los Braganzas, Buenos Aires, Emece Editores, S,A, 1945.
Hegel, Ceorg, Introducción a la historia de la filosofía. Madrid, Libros Albor, 2000.

Iglesias, Francisco, Historia Política de Brasil (15001964), Madrid, Editora Mapfre, 1992.

Lynch, John, América Latina, entre colonia y nación. Barcelona, Critica, 2001.

Mota, Marly, "O Brasil faz 100 anos: a herenca portuguesa em questioa, en, A Experienca a Primera República no Brasil e en Portugal, Coimbra University Press.

Oliveira lima, Manuel, Dom Joao VI no Brasil, Rio de Janeiro, Topbooks, 1996.

.Formación histórica de la nacionalidad brasileira, Madrid, Editorial-América, 1918.

Seckinger, Ron, The Brazilian Monarchy and the South American Republic 1821-1831, Baton Rouge, Louisina State Universioty Press. 1984.

Singer, André, Os sentidos do Iulismo. Reforma gradual e pacto conservador, Sao Paulo, Companhia das Letras, 2011.

Tarquino de Soussa, Octávio, José Bonifacio, Río de Janeiro, Livraria José Olympio, 1974.

Uricochea, Fernando, The Patrimonial Foundations of the Brazilian Bureaucratic State, University of California Press. 1980. 
○

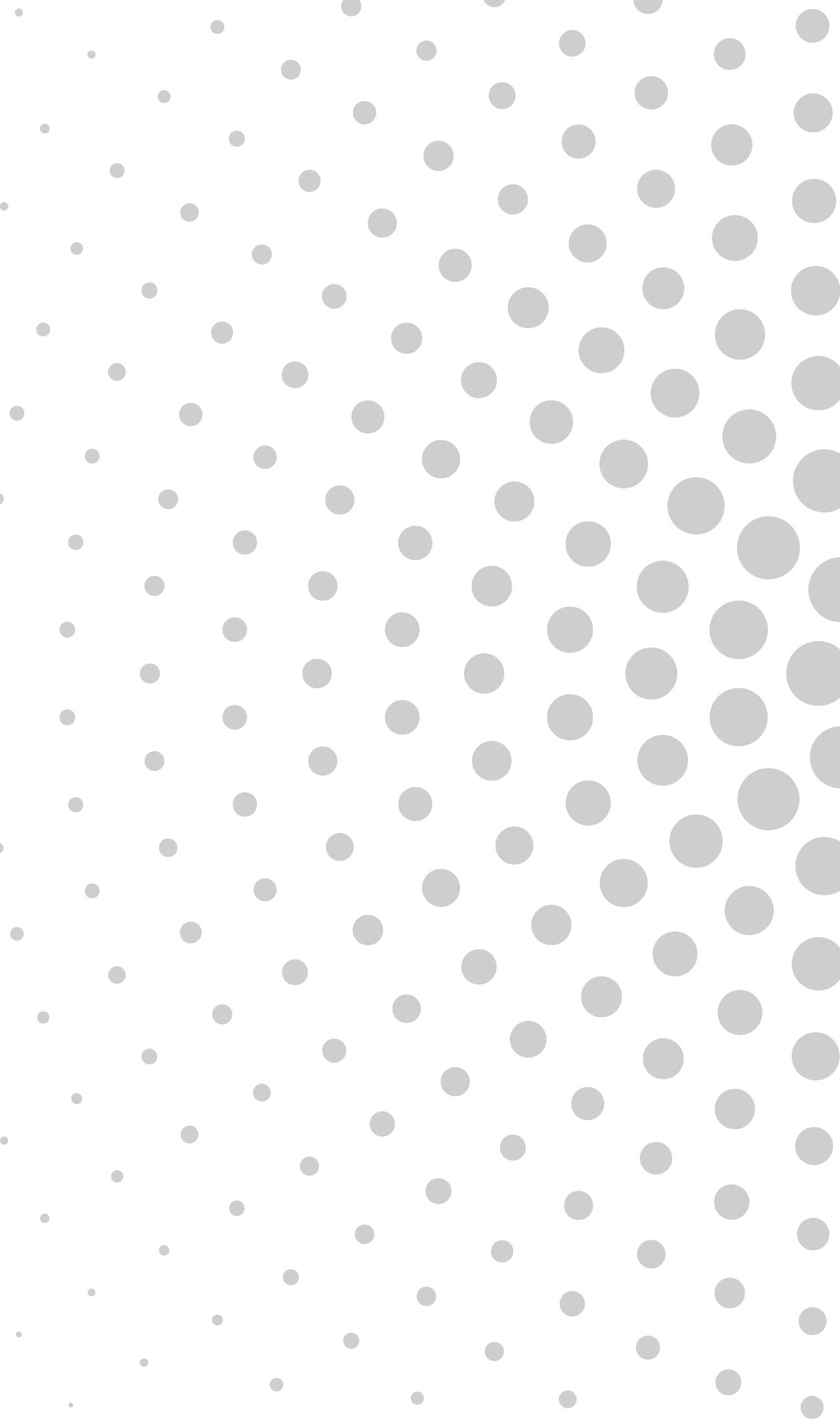

